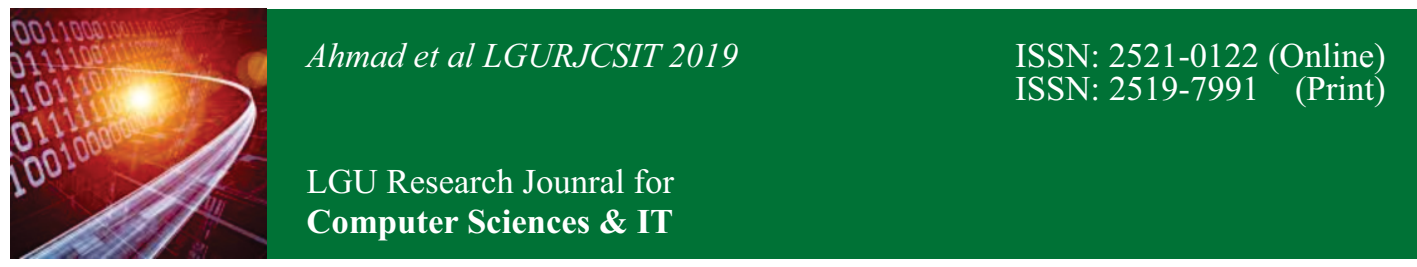

Vol. 3 Issue 4, October - December 2019

\title{
Parallel Data Transmission using New Line Encoding Method
}

Waqas Ahmad ${ }^{1}$, Awais Salman Qazi ${ }^{2}$

${ }^{1}$ Departement of Computer Science \& Information Technology, Lahore Garrison University, Lahore, Pakistan

${ }^{2}$ Department of Computer Science \& Information Technology, Lahore Garrison University, Lahore, Pakistan

Email:waqasahmad@lgu.edu.pk ${ }^{1}$, awais.salman@lgu.edu.pk ${ }^{2}$

\begin{abstract}
:
In our research paper, we have introduced a unique and new line code method/technique that will have the capability to send two different codes in parallel or concurrently using a single line code, and this will be achieved without any major loss in the shape of the signal. In our proposed new line code, the structure of our code is based on two already well-known line codes that have been used many times in the data communication field, both of them have been combined together. We can say that our new line coding method is a hybrid encoding scheme. This line code combines the properties of already existing line code techniques. At the receiver end, we have implemented a very easy and basic separation technique that shall help us to separate the real user codes from the added line code, and this will happen without any disturbance or major loss/distortion. There will be no effect on the signal of input data.
\end{abstract}

Keywords: $\quad$ Line Code, Distortion, Parallel

\section{Introduction}

In computing environments 1 ike telecommunication and networks field, a line coding which is also known as digital baseband, the modulation is selected to be used in digital communication and transmission environment for a purpose of baseband transmission. There are various line coding algorithms that are widely used for digital data transmission in which binary data in the form of one (1) and zero $(0)$, is represented in various digital signaling formats. The same is the case in Pulse Code Modulation signaling [2, 6]. In every encoding method, binary data is sent using a number of rectangular-shaped pulses. The decision of what size of pulses should be used to represent the binary bits one (1) and zero ( 0 ) is normally made on the ground realities using the following considerations i.e. whether DC level is present or absent, efficiency of bandwidth, level of transparency, is it easy to recover clock signal and availability of error detection characteristics
$[2,6]$. There are many line codes available right now, the most popular line codes which are in use worldwide are named as unipolar encoding, polar encoding, bipolar encoding, Manchester encoding and differential Manchester encoding. Brief and precise introduction of popular codes are written as below.

\subsection{Unipolar Encoding:}

In the unipolar line coding, binary bit one (1) is shown by an upward positive voltage, and a binary zero ( 0$)$ is represented by a horizontal line showing zero voltage level. This line coding is the simplest technique. Another name of this technique is on-off keying, termed as $\mathrm{OOK}$, and also known as NRZ (nonreturn to zero) scheme. This coding method is preferred when one symbol is sent much more often as compared to the other. However, this coding technique has some deficiencies as well which are, it is not self-clocking and normalized power is double as compared to the polar NRZ. Therefore, in 
present days, unipolar encoding is not used in the data communication networks field [1].

\subsection{Polar Encoding:}

The polar encoding uses two levels; these levels are either known as polarity or amplitude. It is categorized into three types i.e. NRZ (not return to zero), RZ (return to zero) and biphase. Binary number 1 is represented by the positive voltage level while binary 0 is represented by the negative voltage level [1].

\subsection{Bipolar Encoding:}

Bipolar encoding is a type of RZ line coding, in which we use two non zero values, so the three values are plus (+), minus (-) and zero (0). Bipolar signaling is often called a duobinary signal. Binary bit 1 is represented by positive voltage and then negative voltage while binary bit 0 is represented by a constant zero value. One of the various benefits of bipolar encoding scheme over unipolar is error detection. The term pseudoternary also belongs to bipolar signaling, it refers to the use of three encoded signal levels to represent two-level binary data. Another name is alternate mark inversion $[1,7]$.

\subsection{Manchester Encoding:}

Manchester encoding is a subtype of polar biphase digital coding. In the telecommunication field, Manchester encoding is a type of digital encoding in which binary one (1) is represented by a positive half voltage level then represented by a negative voltage level. Similarly, binary bit (0) is represented by a negative half voltage followed by a positive half voltage level. This line coding technique is different than others in which a bit is represented by +5 volts high state and low state is represented by 0 volts $[5,8]$.

\subsection{Differential Manchester Encoding:}

This encoding scheme is also a subtype of polar biphase encoding. It is the technique in which data and clock signals have been combined together to form a single level 2 data stream. To indicate the logical values, presence or absence of transitions are used in this method. Synchronization is much easier in this method as compared to other encoding methods because the only polarity at the receiver side matters, whether polarity received is the same or different from the previous value. It doesn't matter what logical value is reaching at the receiver side. This line code method is known by many names like Biphase Mark Code, Frequency Modulation, Aiken Biphase, and Conditioned Diphase. In this encoding method, it is not necessary to know the polarity of the transmitted signal. This encoding method has various advantages over already present line code methods like it has robust clock recovery, detection of transmissions is less error-prone and it has zero DC bias. In differential Manchester encoding, there are two versions of the code, in which one version makes the transition for binary bit 0 and no transition for binary bit 1 , similarly, another version of code makes the transition for binary bit 1 and no transition for binary bit $0[6,9]$. Differential Manchester NRZ line code has the advantage of always having a 0 DC value, it doesn't matter what kind of data sequence will be, most importantly it has twice the bandwidth as compared to the unipolar NRZ and polar NRZ code because the size of pulses is half of the width [5].

The waveforms of different line codes are shown in figure 1 below as an example when the stream of data bits is (01101001). All Waveform shapes are different from each other because encoding methods are different in each case. Following encoding, techniques are applied on the given binary data, i.e. Unipolar not return to zero encoding method, polar not return to zero encoding method, unipolar return to zero encoding method, bipolar return to zero encoding method and Manchester encoding method $[1,18]$. We can easily see that stream of bits' representation in the form of waveform is different.

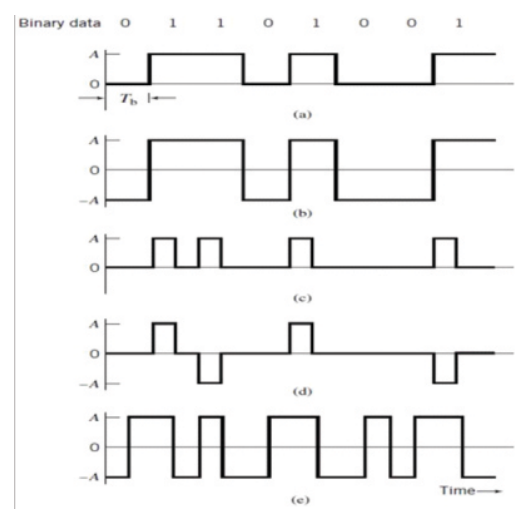

Fig 1. Signal Representation using different encoding techniques 


\section{STRUCTURE OF PROPOSED ENCODING SCHEME}

In our manuscript, we have introduced a unique and different line code technique that can be used to transmit two codes in parallel at the same time using the same bit interval of the onebit period. And particularly in this section, we have tried to show the structure of the proposed new line coding technique based on distinguishing features. However, conventional line code techniques only have the tendency to send one code per bit, which is more time taking and most error-prone [3].

\subsection{Shapes of Signal using New Line Code}

In this manuscript, we have shown how parallel transmission can be done using a new method. We take two bits from two separate codes i.e. the first bit is taken from the first line code and the second bit is taken from another line code. By this coding strategy, we will have four combinations of data bits that are to be transmitted.

Let's suppose we have 2- bits input whose combinations are (00), (01), (10) and (11).

Now if we want to show the above bits by using our proposed line coding scheme, it will be represented in a different way. This representation will be done as follows.

Table No. 1. Data Signals using New Line Coding

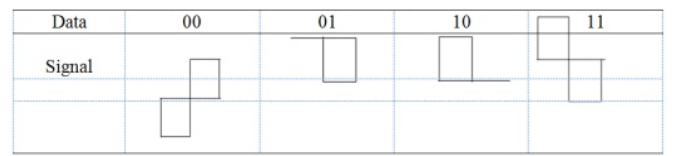

By looking at the above table we can observe the shapes of the signals after they have used new line encoding. The signal is having unique characteristics that are described in bullets below.

- When the data bits are (01), the 0 bit is shown by the horizontal line and the 1 bit is shown by negative pulse downwards.

- When the data bits are (10), the 1 bit is shown by a positive pulse upwards and bit 0 is shown by the horizontal line.

- When the data bits are (00), the first zero is shown by a negative amplitude pulse and the second zero is shown by a positive amplitude pulse.

- When the data bits are (11), the first 1 bit is shown by positive pulse upwards and the second 1 bit is shown by negative pulse downwards.

\subsection{Transmission Strategy}

We have summarized the transmission strategy of our new line code technique. The way binary bits are transferred using new line method is explained in simple steps.

- Two separate codes will be combined together bit by bit in such a way that the first bit belongs to the first code and the second bit belongs to the second code.

- Represent each and every combination of code by its relevant pulse shape and then transmit the signal to the receiver side.

Let us take a look at a scenario of transmitting two separate codes, which will give a clearer idea that how the data is sent using our new line encoding technique. Assume the binary codes are (01010) and (11000) as an example which we want to transmit using new line encoding method. Table 2 shows the two different binary numbers and its converted new line code data. Converted new line data will be transmitted.

Table No. 2. Data encoded using New Line Code

\begin{tabular}{cccccc}
\hline Code 1 & 0 & 1 & 0 & 1 & 0 \\
Code 2 & 1 & 1 & 0 & 0 & 0 \\
Code to transmit & 01 & 11 & 00 & 10 & 00 \\
\hline
\end{tabular}

The signal that will be transmitted using new line coding will look like in the following shape, which is shown in figure 2. The upper wave shows a positive voltage level while the lower wave shows a negative voltage level. The following waveform can also be verified if we take a look at table 1 again.

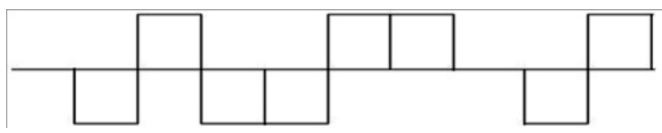

Fig 2. Waveform after using new line coding 


\section{PERFORMANCE OF LINE CODE}

In this section, we have compared our proposed new line code based on performance with all already existing line codes techniques and the parameters we discuss here are selfsynchronization, the density of the power spectral and the decoding algorithm used for new line coding.

\subsection{Self Synchronization}

It is the Line coding technique that will make it possible for the receiver to synchronize the phase of the signal received. For instance, the synchronization of the signal is not done according to the needs, then the decoded signal will be problematic and not reach to the desired optimal differences in terms of the amplitude of different digits in line code. Due to this, there will be huge chances of error in the received signal [15]. Our new line code method is different, and possesses a feature that works on a technique of one-bit transition, therefore our new line code is better than all existing line codes. Our new line code method has features like self-synchronization which is helpful in detecting errors.

\subsection{Relationship of PSD and Normalized Frequency}

The power spectral density (PSD) function exhibits the strength of energy as a function of frequency $[6,16]$. It is worthy to note that PSD of any line code depends on the shapes of pulses and rates that have total correspondence with the digital values. As we already know that our proposed new line coding technique is a combination of two different codes which are Manchester encoding and polar RZ encoding. Therefore, the PSD of our proposed new line coding will be the average of the PSD of two other codes named Manchester Encoding and Polar RZ Encoding [17]. Below figure number 3 shows the PSD of our proposed new line coding technique.

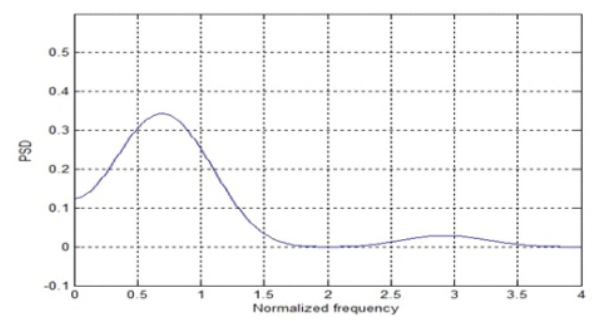

Fig 3. PSD w.r.t Normalized Frequency
In figure 4 below, we have shown the comparison of the PSD of already existing line coding techniques with our proposed new line coding technique in the case of parallel data transmission. We wanted to be sure whether our proposed line encoding technique shows better results in terms of PSD, and the below figure clearly indicates that New Line Coding's PSD is best of all. It can be seen in the below figure that our proposed new line coding technique outclasses all line code techniques and possesses the properties that remaining line coding techniques don't have it. For example, new line code technique outclasses others in terms of smaller DC values and less spectral contents [11, $12]$.

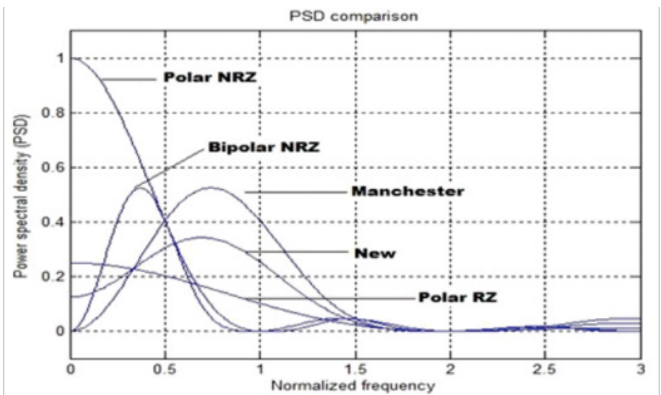

Fig 4. PSD Comparison of Various Encoding Methods

\section{Decoding Algorithm of Proposed New Line Code}

The decoding algorithm used in the new line code technique is very simple and effective, which we have already discussed in previous sections. Once the data is encoded at the transmitter side, it has to be decoded at the receiver side to obtain the real data. Every encoding method has its own way of decoding the transmitted signal. We have used a different way to decode the transmitted signal. Simply, we have to use one-line code for one bit and another line code for upcoming bit, and then combine together. This algorithm is explained in steps as follows.

- In the new line scheme, the code of first bit always refers to the positive side of the new line code while the code of the second bit always refers to the negative side of new line code.

- In the new line scheme, the top upper side if the user input data is binary bit 1 then the positive value will be in the first half of the bit 
period and on remaining half will be negative.

- In the new line scheme, the lower downward side if the user input data is binary bit 1 then the negative value will be in the second half of the bit period and on remaining half will be positive.

\section{Conclusion}

The new line code technique shown in this research paper has shown wonderful performance in terms of clock recovery, phase spectral density, and bandwidth efficiency. All existing conventional line codes have also been checked with these parameters and it is clearly observed that our proposed new line coding technique is far much better than others. None of the existing line coding techniques have the tendency to send two codes concurrently, but the new line code technique can send two codes concurrently, which is a huge plus point. By doing this one could conserve the bandwidth. In a computing environment, it is very necessary that we use an efficient encoding technique. The new Line coding scheme has many advantages over previous encoding techniques. This proposed line coding technique scheme will contribute a lot to the telecommunication and networks field.

\section{REFERENCES}

[1] Khmaies Ouahada, Hendrik Christoffel Ferreira, "Simulation Study of the Performance of Ternary Line Codes under Viterbi Decoding", IEEE Proceedings Communications, Vol. 151, No. 5, November 2004.

[2] Micheal P. Fitz, July 18, 2008, "Fundamentals of Communication Systems", 1st edition, McGraw Hill Education.

[3] Valeriu Munteanu, Daniela Thiceriu, "Information Analysis of the NRZ-M Line Codes and Their Generalization", International Symposium on Signals, Circuits and Systems, Romania, IEEE, 2005.

[4] Zuojian Song and Yoshitaka Takasaki, "Line Coding for Clock Recovery with Minimal Filtering", IEEE Pacific Rim Conference on Communications Computers and Signal Processing, Victoria BC Canada, 2007.
[5] Nikola Alic, "Performance Benefits of Line Coding in the Context of Direct and Coherent Detection", Lasers and Electro Optic Society Annual Meeting-LEOS, November 2008.

[6] Simon Haykin, "Digital Communication Systems, John Wiley \& Sons”, Inc, Feb 2013.

[7] Bernard Sklar, Pabitra Kumar Ray, "Digital Communications Fundamentals and Applications", 2nd Edition, Pearson Education.

[8] M. Bhagyaveni, R. Kalidoss and K. Vishwaksenan, "Introduction to Analog and Digital Communication", River Publishers Series in Communication, Vol. 46, Year 2016.

[9] Simon Haykins, "Communication Systems”, John Wiley \& Sons, 2016.

[10] Dae Young Kim, "Condition for Stable Minimum-Bandwidth Line Codes", IEEE Transaction on Communications, VOL. COM33, NO. 2, Feb 1985.

[11] H. Chung, S. Jeon, "Clock and Data Recovery of an Extended Manchester Code for Pulse Amplitude Modulation", 978-1-46734828-7/12, IEEE 2012.

[12] D. oner, "Criteria for Choosing Line Codes in Data Communication", Istanbul University, Journal of Electrical and Electronics Engineering, Vol 3, No. 2, 2003.

[13] Swati Verma and Rohit Singh, "Multilevel NRZ Coding for Transmission of Digital Signals", International Journal of Advanced Research in Science and Engineering, Vol. No.2, Issue No.9, September 2013.

[14] Nuha A and Abdelrasoul J, "Method of Unipolar Digital to Digital Encoding Data Transmission", Journal of Engineering IOSR, Vol. 04, Issue 03, March 2014.

[15] Glass A Bastaki, "H-Ternary Line Code for Data Transmission", International Conference on Communications, Computer and Power (ICCCP 2001), Sultan Qaboos University, Muscat, Oman, Feb 12-14(2001) 
[16] Antonio Campillo, Patrick Fitzpatrick, Edgar Martinez and R. Pellikaan, "Special Issue Algebraic Coding Theory and Applications", Journal of Symbolic Computation, July 2010.

[17] R. Togneri, C. DeSilva, "Fundamentals of Information Theory and Coding Design", Chapman \& Hall/CRC, 2006.

[18] San Ling and Chaoping Xing, "Coding Theory”, Cambridge University Press, 2004. 